\title{
El uso del factor liberador de hormona de crecimiento (GRF) como prueba de reserva hipofisiaria para hormona de crecimiento $(\mathrm{hGH})\left({ }^{*}\right)$
}

\author{
B.Q. Carmen Campino J, 1; Dr. Ronald Youlton R.2; D. Hugo Pumatino C. 1; \\ T.M. Bernatdo Bruggendieck M. 1 ; Dr. Patricjo Contresas C. 1 \\ Growth hormone releasing factor (GRF) as \\ a test of pituitary reserve of growth homone (hGH)
}

Severad non specific non physiological stimulatory lest are used in the study of pituitary capacity to secretc loGH but none of them is completely reliable. We have used intravenous GRF ( $1 \mu \mathrm{g} \times \mathrm{kg}$ body weight) in 6 shert stature but endocrinologically normal children and w 4 patients with proven hGH deficiency. The maximat plasma hGH concentration reached during the test was $47.4 \pm 15.4 \mathrm{ng} \mathrm{x} \mathrm{ml}$ in the normal children. Three of the hGH deficient patients did not respond to this provocative test; the fourth patient showed a blunted response $(5.3 \mathrm{ng} \times \mathrm{ml})$, suggesting a hypothalamic origin of his deficiency. GRF should be considered a good test for the diagnosis of hGH deficiency and one of the modes of therapy of this disorder in the future.

(Key words: Pituitary reserve, growth hormone, GH releasing factor).

La deficiencia de somatotrofina (hGH) es una de las causas de deterioro progresivo del crecimiento, cuya incidencia se estima en uno de cada 5.000 niños'. La secreción hipofisiaria de hGH es controlada por un doble mecanismo hipotalámico, siendo inhibida por la somatostatina o factor inhibidor (GHIF o SS) y estimulada por el factor liberador de hormona de crecimiento (GRF) ${ }^{2}$. Este último es un péptido de 44 aminoácidos, aislado en 1982 , de un tumor pancreático de un paciente acromegálico sin adenoma hipofisiario y que en la actualidad se obtiene por síntesis química ${ }^{3.4}$. La secreción de hGH es episódica y ocurren 7 a 8 pulsos secretorios en las 24 horas, por lo que una muestra de sangre obtenida al azar raramente permite distinguir al individuo normal de deficiente en $\mathrm{hGH}^{5}$. Para ello se han descrito diversos estímulos, ninguno de los cuales es eficaz en todos los individuos ${ }^{6}$. Considerando el efecto del GRF sobre la secreción de hGH lo hemos utilizado para determinar la capacidad secretoria de esta hormona en sujetos controles y

1. Servicio de Endocrinologia, Hospital Clínico Universidad de Chile.

2. Servicio de Genética, Hospital Clínico Universidad de Chile.

(*) Presentado en Jornadas Chilenas de Endocrinología. Santiago. Chile. Noviembre 1985

Parcialmente financiado Proyecto M-1695-8533 Universidad de Chile. en pacientes con déficit de somatotrofina. La información preliminar de este estudio es objeto de la presente comunicación.

\section{PACIENTES Y METODOS}

Se estudió un grupo de 6 niños ( 2 niñas y 4 varones) de 8 a 13 años de edad, endocrinológicamente normales y que consultaron por talla baja. Simultáneamente con el grupo anterior se estudiaron 4 pacientes con déficit de hGH: una niña de 6 años y 3 varones de 18,20 y 50 años en quínes el diagnóstico se comprobó por los síntomas y signos clínicos característicos y por la no respuesta de la somatotrofina para a lo menos dos de los estímulos tradicionales para provocar liberación de hormona de crecimiento. Los pacientes RV, AV e IM, padecen de una deficiencia selectiva de hGH; MS tiene además, defíciencia de TSH y gonadotrofinas y está en ratamiento con $\mathbf{L}$-tiroxina y testosterona. RV es hija de AV. Algunos de los datos clínicos y de laboratorio de los pacientes $y$ los niños control se presentan en la Tabla 1 .

La relación edad-talla y edad-peso se calculó de acuerdo a las tablas de Tanner ${ }^{7}$. La edad ósea fue determinada de acuerdo al atlas de Greulich y Pyle ${ }^{8}$.

Las pruebas de estimulación para hGH (hipoglicemia insulínica y GRF) efectuaron, previo consentimiento informado, manteniendo una vía venosa permeable. Para todos los estudios se tomaron 2 muestras basales con diferencia de $\mathbf{3 0}$ minutos. Para la hipoglicemia se inyectó insulina corriente $(0,1 \mathrm{U} \times \mathrm{kg}$ de peso e.v.) y se tomaron muestras a $\operatorname{los} 30,60,90$ y 120 minutos para medir glicemia y $h \mathbf{H G}$.

La prueba de GRF se realizó suministrando' $1,0 \mathrm{ug} x$ kg de peso, endovenoso (e.v.) y se tomaron muestras a los $20,40,60,100$ y 120 min para medir $h G H$.

En ambos grupos de niños se realizó en otra oportu. nidad, una infusión e.v. salina durante 150 minutos con 

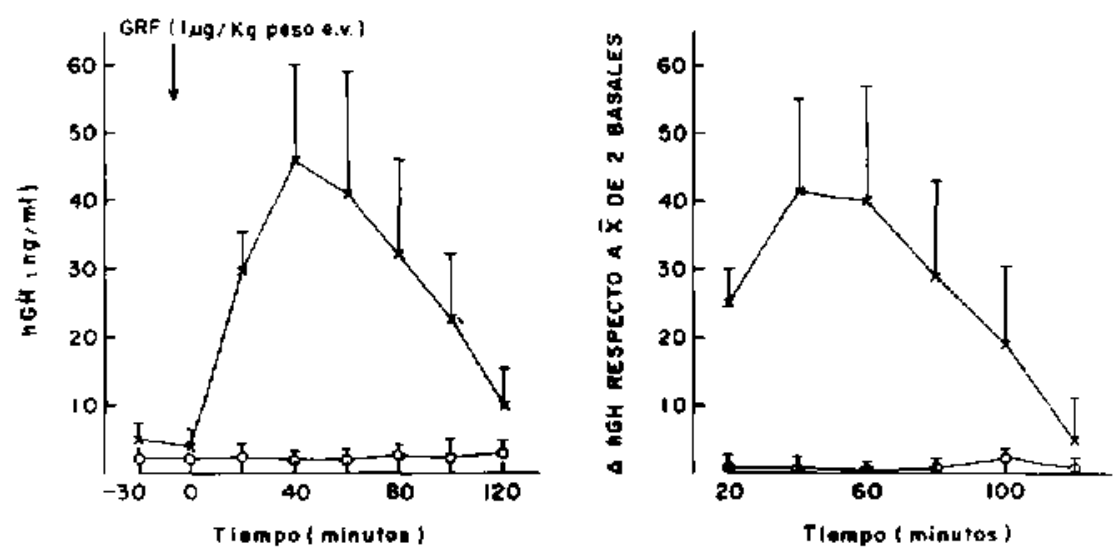

Figura 1. Respuesta de hGH plasmática a GRF en seis sujetos controles. A izquierda: respuesta absoluta. A derecha: Delta sobre el promedio de los dos valores basales $\mathrm{x}-\mathrm{x}$ GRF $0-0$ solución salinạ.

toma de muestras cada 30 minutos para estudio de su secreción espontánea de hGH.

Las determinaciones de T4, TSH y hGH se hicieron por radioinmunoanálisis9. Para estas dos últimas se usaron estándares y anticuerpos donados por el Piturtary Hormone Distribution Program de NLAMDD Bethesda, Maryland U.S.A.

Los niveles de hGH para los distintos estímulos están expresados como ng $x \mathrm{ml} y$ como delta respecto al promedio de 1 los 2 valores basales.

Los resultados están expresados como promedio $y$ error estándar.

\section{RESULTADOS}

La Figura 1 muestra la respuesta de la hGH al GRF en el grupo control. Se observa un significativo aumento detectable a los $20 \mathrm{~min}$. que llega a un máxirno a los $40 \mathrm{~min}$. A pesar de la gran variabilidad en las respuestas todos los individuos alcanzaron niveles sobre $18 \mathrm{ng} \times \mathrm{ml}$ y el delta mínimo fue de $14,7 \mathrm{ng} \mathrm{xml}$. Todos los sujetos toleraron bien la dosjs suministrada.

La respuesta de la somatotrofina a la hipoglicemia insulinica y a GRF en 4 pacientes con déficit de hGH se presenta en la Figura 2. Se observa que ninguno respondió a la hipoglicemia insulínica y sólo vno, el multitrópico, tuvo una respuesta parcial al GRF, alcanzando un valor de $5,3 \mathrm{ng} \times \mathrm{ml}$ a los $60 \mathrm{~min}$. de inyectado el GRF. (Figura 2).

\section{DISCUSION}

Desde el descubrimiento del GRF en 1982, han aparecido. múltiples evidencias que confirman la especificidad del GRF para provocar sólo la liberación de $\mathrm{hGH}^{2}, 10$, sin modificar los niveles de glicemia, ni los de otras hormonas hipofisiarias.

A diferencia de los otros estímulos comúnmente usados en clínica tales como L-Dopa, hipoglicemia por insulina etc., ${ }^{1}$ el GRF es muy bien tolerado y la magnitud de su respuesta, en nuestra experiencia, ha sido superior a la de los estímulos anteriores ${ }^{12}$.

Las publicaciones extranjeras confirman que todos los sujetos normales responden a este estimulo 2.10 , a diferencia de los hasta ahora utilizados que son inespecíficos, no físiológicos y no siempre efectivos, razón por la que se exige una falta de respuesta a no menos de 2 de ellos

Tabla 1.

Antecedentes clínicos y de laboratorio en nin̄os con déficit de hormona de crecimiento y controles.

\begin{tabular}{|c|c|c|c|c|c|c|c|c|c|c|}
\hline & \multicolumn{4}{|c|}{ PACIENTES } & \multirow[b]{2}{*}{ C.Z. } & \multicolumn{5}{|c|}{ CONTROLES } \\
\hline & R.V. & A.V. & I.M. & M.S. & & P.V. & R.G. & C.B.F. & C.E.L. & o.v. \\
\hline Sexo & $\mathrm{F}$ & $\mathbf{M}$ & $\mathbf{M}$ & $\mathbf{M}$ & $\mathbf{F}$ & $\mathrm{F}$ & $\mathbf{M}$ & $M$ & M & $M$ \\
\hline Edad cronológica & 6,5 & 30 & 20 & 18,5 & 11,0 & 8,0 & 9,5 & 13,1 & 13,9 & 11,5 \\
\hline Edad talla & 3 & 10,5 & 9,5 & 10,5 & 9,5 & 6,9 & 7,3 & 10,2 & 9,5 & 9,3 \\
\hline Edad peso & 3,5 & 15 & 11 & 10,5 & 11,0 & 7,0 & 7,0 & 9,8 & 10,5 & 10,3 \\
\hline Edad ósea & 3 & Adulto & 16 & 13,5 & - & 5,3 & 9,5 & - & 11,0 & - \\
\hline $\mathrm{T}_{4}$ ug/dl (VN 4,7-12,5) & 7,2 & 7,2 & 7,2 & 6,7 & 7,7 & 8,0 & 7,7 & 7,7 & 7,4 & 7,1 \\
\hline TSH UUL/ml (VN 7,5) & 4,0 & 4,5 & 3,0 & 1,2 & 3,5 & 3,0 & 1,5 & 1,5 & 1,5 & 5,0 \\
\hline
\end{tabular}


Hipoglicemia

GRF

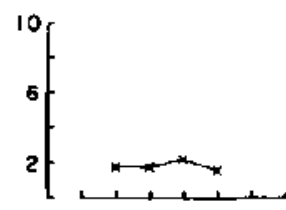

I.M.
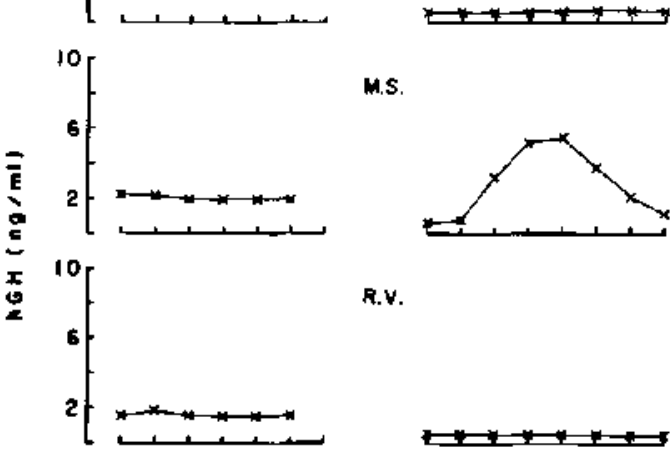

M.S

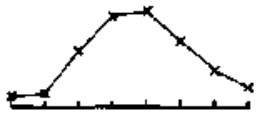

R.v.
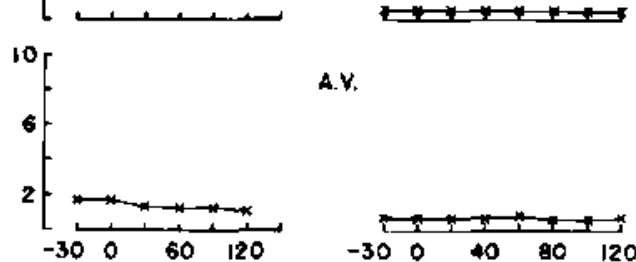

A. V.

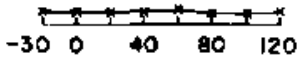

Tiempo (minutos)

Figura 2. Respuesta de hGH plasmática a hipoglicemia insulínica (izquierda) y a GRF (derecha) en cuatto sujetos con deficiencia de hGH.

para establecer el diagnóstico de deficiencia de hGH.

Los 6 niños estudiados como control tuvieron una respuesta de $\mathrm{hCH}$ superior a $18 \mathrm{ngxml}$ y claramente distinguibles de los 4 pacientes con diagnóstico previo de deficjencia de hGH. Tres de estos no respondieron; sólo MS mostró un incremento parcial de su hGH plasmática $(5.3 \mathrm{ng} \mathrm{x} \mathrm{ml})$. Esta respuesta sugiere que su defecto no radica en la hipófisis sino en el mecanismo de síntesis o liberación de su GRF hipotalámico ${ }^{13}$ y es posible que ante estímulos repetidos sus respuestas se acerquen a la normal.

Con una dosis única de GRF como la utilizada en el presente estudio, no podemos asegurar que Ios otros 3 pacientes tengan una falla a nivel de la hipófisis, ya que una glándula no estimulada por arfos podria tener una respuesta más lenta. Sin embargo lo más probable es que en estos casos, con la primera inyección de GRF, exista algún grado de respuesta, aún cuando no se logre que ella sea maxima. No creemos que su falta de respuesta haya sido debida a la cantidad de GRF inyectada, ya que las publicaciones referentes a curvas dosis-respuestas, indican que la liberación de hGH se logra ya a dosis de $0,1 \mu \mathrm{g} \mathrm{x} \mathrm{kg} \mathrm{de} \mathrm{peso}$ e.y. y que dosis superiores a $1,0 \mu \mathrm{g} \times \mathrm{kg}$ de peso e.v. no la magnifican ${ }^{14}$.
La secteción de GRF está modulada por diver. sos estímulos, uno de los cuales es de tipo alfa adrenérgico ${ }^{15}$. Utilizando un agonista alfa adrenérgico como la clonidina, se podria explorar la reserva hipotalámica de $G R F$, lo que actualmente estamos efectuando en el paciente MS.

La utilidad del GRF no sólo reside en el diagnóstico sino también en la terapéutica, ya que permite identificar los pacientes que deben ser tratados con hGH y aquellos que serían susceptibles de ser tratados con GRF cuando sea producido por la industria farmacéutica.

\section{RESUMEN}

Diferentes pruebas inespecíficas de estimulación se emplean para estudiar la capacidad de la hipofisis para secretar hormona del crecimiento, pero ninguno es totalmente confiable. Los autores emplearon factor liberador de hormona del crecimiento ( $1 \mathrm{\mu g} \times \mathrm{kg}$ de peso corporal endovenoso) en 6 niños de talla baja pero sin afecciones endocrinas $y$ en 4 pacientes con deficiencia comprobada de homona del crecimiento. La concen. tración plasmática máxima $(\overline{\mathrm{X}}+\mathrm{DE})$ de la hormona en los niños normales, durante la prueba, fue de $47,4 \pm 15,4 \mathrm{ng} x \mathrm{ml}$. Tres de los cuatro pacientes no mostraron respuesta a la provoca. ción y en el cuarto la concentración plasmática máxima alcanzada fue de $5,3 \mathrm{ng} \times \mathrm{ml}$ sugiriendo un origen hipotalamico para su deficiencia hor. monal. El empleo de factor liberador sería de utilidad en el futuro para el diagnóstica y tratamiento de la deficiencia de hormona del crecimiento.

\section{AGRADECIMIENTOS}

Los autores agradecen al Pifuitary Hormone Distribution Program de NIAMDD, Bethesda, Maryland, el suministro de hormona de crecimiento $y$ de TSH.para yodar.

También queremos agradecer al Dr. Pierre Chatelain $y$ al Labotatorio Clin-Midy el GRF (1-44) para los estudios in vivo.

\section{REFERENCLAS}

1. Schaff-Blass, E., Burstein, S., Rosenfield, R.L.: Advances in diagnosis and treatment of short stature, with special reference to the role of growth hormone. J. Pediatr. 104: 801, 1984.

2. Gómez-Pan, A., Rodriguez-Amao, M.D.: Somatostatin and growth hormone releasing factor: synthesis, location, metabolism and function. Clin. Endocrinol. Metab. 12: 469, 1983.

3. Guillemin, R., Brazeat, P., Bohlen, P.: Growth hormone-releasin factor from a pancreatic tumor that caused acromegaly. Science 218: $585,1982$.

4. Rivier, J., Spiess, J., Thorner, M., Vale, W.: Characterization of a growth hormone-releasing factor from a human pancreatic islet tumour. Nature 300 : $276,1982$. 
5. Daughaday, $\boldsymbol{W}, \boldsymbol{H}_{1}$ : The adenohypoplyssis Williams RH (Ed) Textbook of Endocrinology (5a Edición) W.G. Saunders Company 1974: 31-79.

6. Youlfon, R. Kaplan, S.L., Grumbach, M.M.: Growth and growth hormone IV. Limitations of the growth hormone response to insulin and arginine and of immunoreactive insulin response to arginine in the ašsessment of growth hormone deficiency in children. Pediatrics 43: 989, 1969.

7. Tonner, J.M., Whitehouse, R.H.: Longitudinal standards for height, weight, height velocity, weight velocity and stages of puberty. Arch. Dis. Child. 51 : $170,1976$.

8. Greulich. W.W., Pyle, I.S.: Radiographic atlas of skeletal development of the hand and wrist. Second. ed. Stanford, California, 1951.

9. Campino, C., Pumarino, H.: Hormona de crecimiento. I Consigeraciones fisiológicas y bioquímicas. Rey, Med. Chil 110: 43, 1982.

10. Gehto, M.C., Pescovitz, O. Cassorla, F., Loriaux, L., Merriom, G.: Effects of a growth hormone releasing factor in man. J. Clin. Endocrinol. Metab. 57: 674,1983 .
11. Lin, T., Tucci, J.R.: Provocative tests of growthhormone release. A comparison of results with seven stimuli. Ann. Intern. Med. 80: 464, 1974.

12. Campino, $C_{1}$ Pumarino, $H_{.}$Observaciones no publicadas.

13. Schrock, E.A., Lusting, R.H., Rosenthal, S.M., Kaplan, S., Grumbach, M.M.: Effect of growth hormone (GH) - releasing hormone (GRH) on plasma $\mathbf{G H}$ in relation to magnitude and duration of $G H$ deficiency in 26 children and adults with isolated $\mathrm{GH}$ deficiency or multiple pituitary deficiencies; evidence for hypothalamic GRF deficiency. J. Clin, Endocrinol. Metab. 58: 1043, 1984.

14. Thorner, M.O., Vance, M.L. Evans, W.S., Blizzard, R., Rogol. A.D., Kaiser, D.L., Furlanetro, R., Frohmon, L.A., Rivier, J., Vale, W.: Clinical implication of growth hormone releasing factor. Bizollon (ed) Monoclonal antibodies and new tsends in immunoassays. Elsevier Science publishers. Amsterdam, 1984: 265-275.

15. Carestrom, $K .:$ Effects of clonidine on polypeptide and sterioid hormone levels in man. Acta Obstet. Gynecol. Scand. Suppl. 132: 33, 1985. 